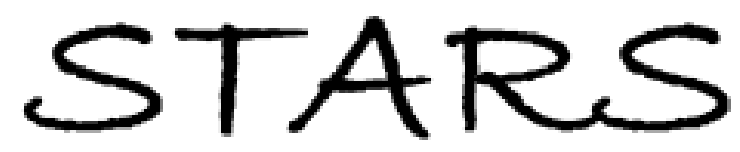

University of Central Florida

STARS

$1-1-2014$

\title{
General rotating quantum vortex filaments in the low-temperature Svistunov model of the local induction approximation
}

Robert A. Van Gorder

University of Central Florida

Find similar works at: https://stars.library.ucf.edu/facultybib2010 University of Central Florida Libraries http://library.ucf.edu

This Article is brought to you for free and open access by the Faculty Bibliography at STARS. It has been accepted for inclusion in Faculty Bibliography 2010 s by an authorized administrator of STARS. For more information, please contact STARS@ucf.edu.

\section{Recommended Citation}

Van Gorder, Robert A., "General rotating quantum vortex filaments in the low-temperature Svistunov model of the local induction approximation" (2014). Faculty Bibliography 2010s. 6214.

https://stars.library.ucf.edu/facultybib2010/6214

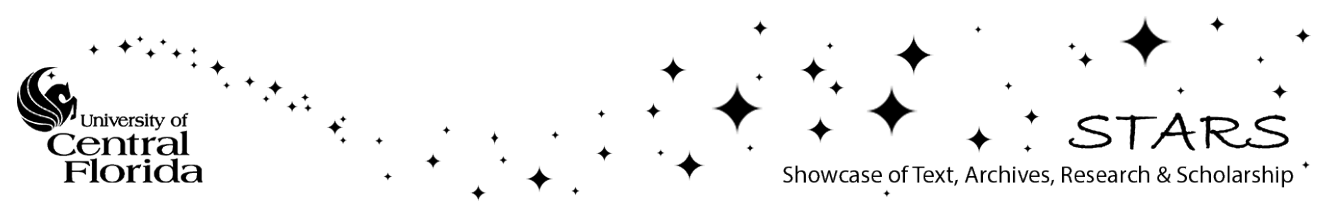




\section{General rotating quantum vortex filaments in the low-temperature Svistunov model of the local induction approximation}

Robert A. Van Gorder

Citation: Physics of Fluids 26, 065105 (2014); doi: 10.1063/1.4883749

View online: https://doi.org/10.1063/1.4883749

View Table of Contents: http://aip.scitation.org/toc/phf/26/6

Published by the American Institute of Physics

\section{Articles you may be interested in}

Helical waves on a vortex filament

American Journal of Physics 73, 563 (2005); 10.1119/1.1873892

Motion of a helical vortex filament in superfluid ${ }^{4} \mathrm{He}$ under the extrinsic form of the local induction approximation Physics of Fluids 25, 085101 (2013); 10.1063/1.4816639

Motion of isolated open vortex filaments evolving under the truncated local induction approximation Physics of Fluids 29, 115105 (2017); 10.1063/1.5005113

Non-local dynamics governing the self-induced motion of a planar vortex filament Physics of Fluids 27, 065105 (2015); 10.1063/1.4922171

Translation of waves along quantum vortex filaments in the low-temperature two-dimensional local induction approximation

Physics of Fluids 27, 095104 (2015); 10.1063/1.4930226

Physical interpretation of certain invariants for vortex filament motion under LIA

Physics of Fluids A: Fluid Dynamics 4, 938 (1992); 10.1063/1.858274

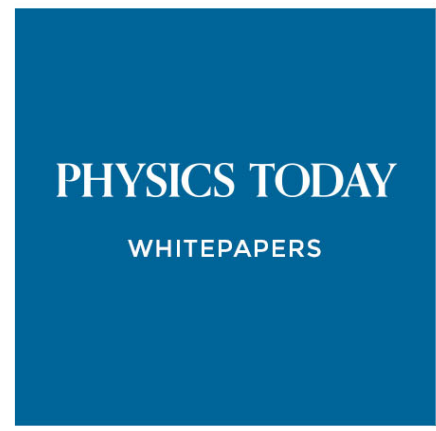

ADVANCED LIGHT CURE ADHESIVES

Take a closer look at what these environmentally friendly adhesive systems can do

\section{READ NOW}

PRESENTED BY Q MASTERBOND 


\title{
General rotating quantum vortex filaments in the low-temperature Svistunov model of the local induction approximation
}

\author{
Robert A. Van Gorder ${ }^{a)}$ \\ Department of Mathematics, University of Central Florida, Orlando, \\ Florida 32816-1364, USA
}

(Received 26 January 2014; accepted 4 June 2014; published online 17 June 2014)

\begin{abstract}
In his study of superfluid turbulence in the low-temperature limit, Svistunov ["Superfluid turbulence in the low-temperature limit," Phys. Rev. B 52, 3647 (1995)] derived a Hamiltonian equation for the self-induced motion of a vortex filament. Under the local induction approximation (LIA), the Svistunov formulation is equivalent to a nonlinear dispersive partial differential equation. In this paper, we consider a family of rotating vortex filament solutions for the LIA reduction of the Svistunov formulation, which we refer to as the 2D LIA (since it permits a potential formulation in terms of two of the three Cartesian coordinates). This class of solutions holds the well-known Hasimoto-type planar vortex filament [H. Hasimoto, "Motion of a vortex filament and its relation to elastica," J. Phys. Soc. Jpn. 31, 293 (1971)] as one reduction and helical solutions as another. More generally, we obtain solutions which are periodic in the space variable. A systematic analytical study of the behavior of such solutions is carried out. In the case where vortex filaments have small deviations from the axis of rotation, closed analytical forms of the filament solutions are given. A variety of numerical simulations are provided to demonstrate the wide range of rotating filament behaviors possible. Doing so, we are able to determine a number of vortex filament structures not previously studied. We find that the solution structure progresses from planar to helical, and then to more intricate and complex filament structures, possibly indicating the onset of superfluid turbulence. (ㄷ) 2014 AIP Publishing LLC. [http://dx.doi.org/10.1063/1.4883749]
\end{abstract}

\section{INTRODUCTION}

A vortex in the reference frame moving with the superfluid was given by Hall and Vinen. ${ }^{1,2}$ (This model is also referred to as the HVBK model, or Hall-Vinen-Bekarevich-Khalatnikov model. See Bekarevich and Khalatnikov. ${ }^{3}$ ) For a quantized vortex filament, the dynamics are taken to be those given by the Biot-Savart law, which is often approximated by a local induction approximation (LIA). Schwarz ${ }^{4}$ obtained a type of quantum LIA which takes mutual friction and interaction with the normal fluid into account, obtaining

$$
\mathbf{v}=\gamma \kappa \mathbf{t} \times \mathbf{n}+\alpha \mathbf{t} \times(\mathbf{U}-\gamma \kappa \mathbf{t} \times \mathbf{n})-\alpha^{\prime} \mathbf{t} \times(\mathbf{t} \times(\mathbf{U}-\gamma \kappa \mathbf{t} \times \mathbf{n})) .
$$

Here $\mathbf{U}$ is the dimensionless normal fluid velocity, $\mathbf{t}$ and $\mathbf{n}$ are the unit tangent and unit normal vectors to the vortex filament, $\kappa$ is the local curvature, $\gamma=\Gamma \ln \left(c / \hat{\kappa} a_{0}\right)$ is a dimensionless composite parameter $(\Gamma$ is the dimensionless quantum of circulation, $c$ is a scaling factor of order unity with units $\mathrm{cm}, a_{0} \approx 1.3 \times 10^{-8} \mathrm{~cm}$ is the effective core radius of the vortex, $\hat{\kappa}$ is the average curvature), $\alpha$ and $\alpha^{\prime}$ are dimensionless friction coefficients which are small (except near the $\lambda$-point; for reference, the $\lambda$-point is the temperature (which at atmospheric pressure is $\approx 2.17 \mathrm{~K}$ ) below which normal

\footnotetext{
a) Author to whom correspondence should be addressed. Electronic mail: rav@knights.ucf.edu
} 
fluid helium transitions to superfluid helium ${ }^{5}$ ). Regarding reasonable values of $\alpha$ and $\alpha^{\prime}$, Table I of Schwarz ${ }^{4}$ shows that at temperature $T=1 \mathrm{~K}$ we have $\alpha=0.006$ and $\alpha^{\prime}=0.003$, while at temperature $T=1.5 \mathrm{~K}$ we have $\alpha=0.073$ and $\alpha^{\prime}=0.018$. Thus, it makes sense to consider these friction terms as small parameters.

In the $\alpha, \alpha^{\prime} \rightarrow 0$ limit (the zero-temperature limit), the motion of vortex lines is described by the standard Biot-Savart law:

$$
\frac{d \mathbf{r}}{d t}=\frac{\gamma}{4 \pi} \int \frac{\left(\mathbf{r}_{0}-\mathbf{r}\right) \times d \mathbf{r}_{0}}{\left|\mathbf{r}_{0}-\mathbf{r}\right|}
$$

Often the Biot-Savart law (2) is replaced by the LIA. In this case, self-induced velocity of a vortex filament is approximated by ${ }^{6,7}$

$$
\mathbf{v}=\gamma \kappa \mathbf{t} \times \mathbf{n},
$$

where $\mathbf{t}$ and $\mathbf{n}$ are unit tangent and unit normal vectors to the vortex filament, respectively, $\kappa$ is the quantized curvature and $\gamma$ is the strength of the vortex filament. Hasimoto ${ }^{8}$ obtained a 1-soliton solution of the LIA in the curvature-torsion frame. Exact stationary solutions to the LIA in extrinsic coordinate space have been discussed by $\mathrm{Kida}^{9}$ in the case of torus knots, planar solutions, and helices; some of these solutions are given by elliptic integrals.

Hasimoto ${ }^{10}$ considered a planar vortex filament in the curvature-torsion frame of reference. This influential and often cited paper demonstrates the relation between the curvature of a vortex filament and elastica. Such a solution was also considered by Kida, ${ }^{9}$ who obtained results in terms of elliptic integrals in the moving (time-dependent) arc length coordinate frame, with stability results for some filaments in this framework later provided. ${ }^{11}$ Fukumoto $^{12}$ considered the influence of background flows on such stationary states. For the Cartesian frame, some preliminary results were determined in Van Gorder, though only some special solutions were given. There is an alternate formulation, given by Umeki, ${ }^{13,14}$ which provides the LIA in an arc-length coordinate frame. The Hasimoto filament can be determined exactly in this frame (as is also true of the curvature-torsion frame), and the results were worked out by Van Gorder. ${ }^{15}$ Small amplitude space-periodic solutions of planar type were obtained through a multiple scales analysis. ${ }^{16}$ Such solutions are valid in the small-amplitude regime when the nonlinearity becomes sufficiently weak, though solutions break down after that.

In the present paper, we shall be interested in generalized rotating vortex filaments. In order to describe such vortex filaments, we will employ the form of the LIA described in Boffetta et al. ${ }^{17}$ (which we refer to as the 2D LIA, for reasons outlined later) and derived from the Svistunov model. ${ }^{18}$ We show that the model contains both planar and helical filaments (those most often studied in the literature), and that these are really two narrow special cases of rotating planar vortex filaments. In particular, when the solutions have constant amplitude and space-variable phase, they correspond to the helical filaments of Sonin. ${ }^{19}$ Meanwhile, when the phase is constant in space, and the amplitude varies, we recover the planar vortex filaments of Hasimoto type. Each of these solutions is rather narrow, and in general we will have solutions in between these two extremes.

The structure of the paper is as follows. In Sec. II, we provide a formulation of the 2D LIA of Boffetta et al. ${ }^{17}$ We demonstrate why this model is useful for studying non-planar filaments (such as filaments which may exhibit large spatial changes). Section III constitutes an analytical and numerical study of the generalized rotating filaments. First, we determine the system of nonlinear differential equations governing the structure of such a vortex filament. We show that, in general, the phase and amplitude of such a filament are strongly coupled. The change in the phase can be given strictly in terms of the amplitude, which allows us to write one equation for the amplitude which itself depends on two parameters. Studying this equation, we demonstrate the existence of space-periodic filament solutions (in particular, filaments that are periodic in the $y$ and $z$ components, $y(x+T, t)=y(x, t)$ and $z(x+T, t)=z(x, t))$. We show that the period $T$ can be calculated in terms of the model parameters. While the complexity of the differential equation governing the amplitude prevents exact solutions, we can obtain approximate solutions which are perturbative in nature. One of these types of solutions is like the planar filament, whereas the other is quite distinct. Finally, numerical solutions are provided for a variety of cases, in order to demonstrate the range of solutions possible. 
The results indicate a wide variety of behaviors not previously demonstrated mathematically, which hold both helical and planar solutions are rather narrow special cases. In some of the more exotic solutions, we expect degeneracy into turbulence to occur. Hence, some of the solutions discussed here may be useful in the study of the onset of superfluid turbulence. So, these solutions are not a minor generalization of the exact planar or helical filament solutions already known. Rather, consideration of this more general formulation shows us that there are a wide variety of behaviors possible for rotating filaments in the Svistunov model under the LIA.

\section{FORMULATION}

First we consider the LIA (3) directly. Let us assume $\mathbf{r}(x, t)=\left(r_{1}(x, t), r_{2}(x, t), r_{3}(x, t)\right)$, where the $r_{k}$ 's are functions to be determined by the LIA. Calculating $\mathbf{t}$ and $\kappa \mathbf{n}$ and taking the cross product, we obtain the PDE system:

$$
\begin{aligned}
& \left(r_{1}\right)_{t}=\gamma\left(r_{2}\right)_{x}^{2}\left(\frac{\left(r_{3}\right)_{x}}{\left(r_{2}\right)_{x}}\right)_{x}\left(\left(r_{1}\right)_{x}^{2}+\left(r_{2}\right)_{x}^{2}+\left(r_{3}\right)_{x}^{2}\right)^{-3 / 2}, \\
& \left(r_{2}\right)_{t}=\gamma\left(r_{3}\right)_{x}^{2}\left(\frac{\left(r_{1}\right)_{x}}{\left(r_{3}\right)_{x}}\right)_{x}\left(\left(r_{1}\right)_{x}^{2}+\left(r_{2}\right)_{x}^{2}+\left(r_{3}\right)_{x}^{2}\right)^{-3 / 2}, \\
& \left(r_{3}\right)_{t}=\gamma\left(r_{1}\right)_{x}^{2}\left(\frac{\left(r_{2}\right)_{x}}{\left(r_{1}\right)_{x}}\right)_{x}\left(\left(r_{1}\right)_{x}^{2}+\left(r_{2}\right)_{x}^{2}+\left(r_{3}\right)_{x}^{2}\right)^{-3 / 2} .
\end{aligned}
$$

If the filament is aligned along the $x$-axis and includes a translational velocity term (which means that the waves along a vortex filament are permitted to move along the reference axis, in addition to the rotational motion), we take $r_{1}(x, t)=x+\beta t$. In this case, we identify $r_{2}$ and $r_{3}$ with the $y$ and $z$ axes, as $r_{2}(x, t)=y(x, t)$ and $r_{3}(x, t)=z(x, t)$, respectively. As a result, the system (4)-(6) becomes

$$
\begin{gathered}
\beta=\gamma \frac{y_{x} z_{x x}-z_{x} y_{x x}}{\left(1+y_{x}^{2}+z_{x}^{2}\right)^{3 / 2}}, \\
y_{t}=-\frac{\gamma z_{x x}}{\left(1+y_{x}^{2}+z_{x}^{2}\right)^{3 / 2}}, \quad z_{t}=\frac{\gamma y_{x x}}{\left(1+y_{x}^{2}+z_{x}^{2}\right)^{3 / 2}} .
\end{gathered}
$$

From (8), it makes sense to consider a potential function $\Phi(x, t)=y(x, t)+i z(x, t)$, which would put (7) and (8) into the form:

$$
\begin{gathered}
\beta=\frac{\gamma}{2 i} \frac{\Phi_{x}^{*} \Phi_{x x}-\Phi_{x} \Phi_{x x}^{*}}{\left(1+\left|\Phi_{x}\right|^{2}\right)^{3 / 2}}, \\
i \Phi_{t}+\gamma \frac{\Phi_{x x}}{\left(1+\left|\Phi_{x}\right|^{2}\right)^{3 / 2}}=0 .
\end{gathered}
$$

The best way to understand these conditions would be that (10) gives a potential formulation of the LIA provided that the consistency condition (10) is satisfied. When $\beta=0$, there is no drift. In certain situations, it may suffice to find a solution $\Phi$ to (10) such that the right hand side of (9) is very small (though not zero), which results in an approximate solution to the LIA.

The derivation outlined above has been used in many studies, as it is useful when either the amplitude or wave-number are small. Shivamoggi and van $\mathrm{Heijst}^{20}{ }_{\text {reformulated the Da Rios-Betchov }}$ equations in the extrinsic vortex filament coordinate space and were able to find an exact solution to an approximate equation governing a localized stationary solution. In doing so, they re-derived the Cartesian form of the LIA (refereed to as the extrinsic form of the LIA), and obtained a truncated approximation - assuming a very small amplitude. Van Gorder ${ }^{21,22}$ employed this formulation to study a number of special case solutions. Limitations and benefits to this type of formulation were recently a topic of discussion in the sequence, ${ }^{23-25}$ where (for the helical case) the direct approach using (10) (and extensions to the quantum case, where $\alpha, \alpha^{\prime} \neq 0$ ) is most useful when perturbations along filaments are of sufficient bounded variation. Of course, if $\Phi_{x}^{*} \Phi_{x x}-\Phi_{x} \Phi_{x x}^{*}$ is a constant, then we can calculate a parameter $\beta$ which takes care of the translation. For more complicated scenarios, 
when $\Phi_{x}^{*} \Phi_{x x}-\Phi_{x} \Phi_{x x}^{*}$ is not a constant in $x$, the solution of (10) will be an approximation to the LIA, rather than an exact solution.

In the case of a purely planar vortex filament, $\Phi(x, t)=e^{-i t} \phi(x)$, where $\phi(x)$ is real-valued. In this case, $\Phi_{x}^{*} \Phi_{x x}-\Phi_{x} \Phi_{x x}^{*} \equiv 0$, so there is no drift, hence (10) is exactly equivalent to the LIA. In the case where $\Phi_{x}^{*} \Phi_{x x}-\Phi_{x} \Phi_{x x}^{*}$ is small, though not identically zero, (10) is still a reasonably good approximation to the LIA (which itself is an approximation to the filly non-local vortex filament dynamics). However, in the case where $\Phi_{x}^{*} \Phi_{x x}-\Phi_{x} \Phi_{x x}^{*}$ is not small, we evidently need a different formulation, as a solution to (10) will not adequately approximate a solution of the LIA. This formulation should somehow involve a term of the form $\Phi_{x}^{*} \Phi_{x x}-\Phi_{x} \Phi_{x x}^{*}$, and should collapse down to (10) when the term $\Phi_{x}^{*} \Phi_{x x}-\Phi_{x} \Phi_{x x}^{*}$ vanishes. When this term does vanish, there is no translation of the waves along the filament, and the only motion is rotation.

As it turns out, the formulation of Boffetta $e t$ al.,${ }^{17}$ derived in a completely different fashion, shall be of use to us here. In the context of superfluids in the low-temperature limit (i.e., in the absence of superfluid friction parameters), the Cartesian form of the LIA was previously derived by Svistunov in $1995,{ }^{18}$ where the Cartesian representation of the LIA dynamics is represented as a Hamiltonian system for a single periodic vortex line along one axis. Introducing potential $\Psi(x, t)$ $=y(x, t)+i z(x, t)$, Svistunov showed that the Biot-Savart law (2) could be written in Hamiltonian form

$$
i \Psi_{t}=\frac{\delta}{\delta \Psi^{*}} H[w]
$$

where

$$
H[\Psi]=\frac{\kappa}{4 \pi} \iint \frac{1+\operatorname{Re}\left[\Psi_{x}^{*}\left(x_{1}\right) \Psi_{x}\left(x_{2}\right)\right]}{\sqrt{\left(x_{1}-x_{2}\right)^{2}+\left|\Psi\left(x_{1}\right)-\Psi\left(x_{2}\right)\right|^{2}}} d x_{1} d x_{2} .
$$

This is a type of 2D Biot-Savart law. Introducing a cutoff at the vortex filament radius $a<\left|\mathbf{r}-\mathbf{r}_{0}\right|$, the Hamiltonian reduces to the LIA,

$$
\hat{H}[\Psi]=2 \frac{\kappa}{4 \pi} \ln (\ell / a) \int \sqrt{1+\left|\Psi_{x}\left(x_{1}\right)\right|^{2}} d x_{1}=\kappa \gamma L[w],
$$

where $\gamma=\frac{1}{2 \pi} \ln (\ell / a)$ is the filament strength, $\ell$ is a characteristic length scale, and $L[w]$ is the total vortex line length. So, under the LIA, the Hamiltonian is just a scaling of the total vortex length. In Eq. (4) of Boffetta et al. ${ }^{17}$ it was shown that the equation of motion for this Hamiltonian becomes

$$
i \Psi_{t}+\left(\frac{\Psi_{x}}{\sqrt{1+\left|\Psi_{x}\right|^{2}}}\right)_{x}=0,
$$

whereas here we have used a different scaling of $t$ to remove a factor of two from (14). It shall be this equation that we are interested in. Symmetry properties of this model have been discussed by Sonin. ${ }^{26}$ Helical waves from the Cartesian form of the LIA (14) were considered by Sonin. ${ }^{19}$ For more on such Hamiltonian formulations, see Goldstein and Petrich, ${ }^{27}$ Langer and Singer, ${ }^{28}$ and references therein. Fukumoto and Miyajima ${ }^{29}$ construct an integrable hierarchy for the localized induction equation, and determine Kida-type solutions. One area of interest is vortex line reconnections. While a simplification of the full Biot-Savart law, which neglects global behavior in favor of local behavior, the LIA has been shown to admit solutions which do permit self-crossings, ${ }^{4,16,18}$ so it can qualitatively describe vortex line reconnection events in strong turbulence. In contrast, the LIA is not very useful for the study of weak Kelvin wave turbulence, since resonant wave interactions are not present in the simplified LIA model. ${ }^{17}$

Performing the differentiation in $x$ on the second term in (14), we obtain exactly the type of formulation needed:

$$
i \Psi_{t}+\gamma \frac{\Psi_{x x}}{\left(1+\left|\Psi_{x}\right|^{2}\right)^{3 / 2}}+\frac{\gamma}{2} \frac{\Psi_{x}\left(\Psi_{x}^{*} \Psi_{x x}-\Psi_{x} \Psi_{x x}^{*}\right)}{\left(1+\left|\Psi_{x}\right|^{2}\right)^{3 / 2}}=0 .
$$


Note that when $\Psi_{x}^{*} \Psi_{x x}-\Psi_{x} \Psi_{x x}^{*} \rightarrow 0$, (15) reduces to (10) as required (so it accounts for the planar case perfectly). On the other hand, (15) also accounts for situations where $\Psi_{x}^{*} \Psi_{x x}-\Psi_{x} \Psi_{x x}^{*}$ is large. Therefore, this equation is ideal for studying non-planar rotating vortex filaments.

There are a number of known specific solutions forms to the LIA in both classical and quantum settings. Exact solution forms consist of closed vortex rings and knots, ${ }^{6,30-33}$ or loops due to self-intersection ${ }^{4,16,18}$ and the various open filament solutions which consist of helical ${ }^{19,23}$ and planar filaments. ${ }^{9}, 10,15,16$ Another type of filament solution would be the self-similar solutions of the LIA. ${ }^{34-36}$ Solitons have also been found on vortex filaments. ${ }^{8,14,37}$ The present results are an extension of the helical and planar filaments. While both filaments appear essentially distinct in form, they are actually two members of a more general family of rotating vortex filament solutions of the LIA. We find many other members of this family, including filaments which lie between the planar and helical filaments (and which have attributes of both) and also filament solutions occurring at larger amplitudes which take on more complicated forms. However, all of the purely rotating filaments described here do exhibit certain symmetries and therefore show regularity in some sense. In what follows, we shall study such filaments analytically and numerically.

\section{ANALYTICAL AND NUMERICAL PROPERTIES OF THE ROTATING FILAMENT SOLUTIONS}

We first outline properties of a general rotating filament under (14). Later, we shall examine more explicit properties of such filaments, in order to construct analytical or numerical solutions. To model a rotating filament, we assume a solution of the form:

$$
\Psi(x, t)=e^{-\gamma i t} \psi(x) .
$$

In general, $\psi$ must be complex-valued (in order to capture all possible physical behaviors). This class of solution can describe a planar vortex filament when $\psi$ is real-valued; however, when $\psi$ is permitted to be complex-valued, a far wider range of solutions is possible. The Hasimoto-type solutions derived previously move without change in form. ${ }^{9}$ This is in direct analogy to the solutions originally obtained by Hasimoto. ${ }^{10}$ However, if we relax one condition, namely, that $\psi$ be a realvalued function, we can obtain a more general class of solutions. Such solutions will correspond to bending or twisting of the Hasimoto solutions.

Writing $\psi$ in polar form will be more useful, so we consider functions real-valued $R(x)$ and $\Theta(x)$ such that $\psi(x)=R(x) \exp (i \Theta(x))$. Once such a solution is known, then by the definition of $\Psi(x$, $t$ ) we shall have the vortex filament:

$$
\begin{aligned}
\mathbf{r}(x, t) & =(x, y(x, t), z(x, t))=\left(x, \operatorname{Re}\left[e^{-i \gamma t} \psi(x)\right], \operatorname{Im}\left[e^{-i \gamma t} \psi(x)\right]\right) \\
& =(x, R(x) \cos (i\{\Theta(x)-\gamma t\}), R(x) \sin (i\{\Theta(x)-\gamma t\})) .
\end{aligned}
$$

Equation (15) then gives

$$
R+\frac{R^{\prime \prime}-R \Theta^{\prime 2}+i\left(2 R^{\prime} \Theta^{\prime}+R \Theta^{\prime \prime}\right)}{\left(1+R^{\prime 2}+R^{2} \Theta^{\prime 2}\right)^{3 / 2}}=0 .
$$

Since $R$ and $\Theta$ are real-valued functions, we can separate real and imaginary parts. We obtain the real-valued system:

$$
R+\frac{R^{\prime \prime}-R \Theta^{\prime 2}}{\left(1+R^{\prime 2}+R^{2} \Theta^{\prime 2}\right)^{3 / 2}}=0 \quad \text { and } \quad 2 R^{\prime} \Theta^{\prime}+R \Theta^{\prime \prime}=0 .
$$

Solving the latter for $\Theta^{\prime}$, we find $\Theta^{\prime}(x)=\Theta_{1} R^{-2}(x)$ where $\Theta_{1}$ is a constant of integration. If $\Theta_{1}$ $=0$, we effectively have a reduction to the real-valued case considered when studying a planar vortex filament moving without change in form. In such a case, one has

$$
R+\frac{R^{\prime \prime}}{\left(1+R^{\prime 2}\right)^{3 / 2}}=0,
$$


which is exactly what one gets from (10) in the case of a planar filament. This directly gives the Hasimoto planar filament in the Cartesian frame of reference. Various properties of these planar filaments have been discussed previously. ${ }^{16,22}$ On the other hand, when $R(x)$ is constant, say $R(x)$ $=R_{0}$, we obtain a complex exponential solution corresponding to a solution of the form $\Theta(x)=\Theta_{1} x$ $+\Theta_{0}: \Psi(x, t)=R_{0} \exp \left(i\left[\Theta_{1} x+\Theta_{0}-t\right]\right)$. When placed back into (17), this gives a helical solution. Hence, we may recover the helical solutions of Ref. 19 in the constant $R(x)$ case. We remark that in the case of the full Hall-Vinen model (1) with non-zero friction parameters $\alpha>0$ and $\alpha^{\prime}>0$, the motions of purely helical vortex filaments have been described recently by Van Gorder, ${ }^{23}$ although the model considered there used the form of the LIA corresponding to (10) in the case where $A k$ is sufficiently small, and thus neglected any translational effects.

Both the planar and helical filaments are narrow special cases. For the more general case where $\Theta_{1} \neq 0$ and $R$ is not constant, the first equation in (19) becomes

$$
R+\frac{R^{\prime \prime}-\Theta_{1}^{2} R^{-3}}{\left(1+R^{\prime 2}+\Theta_{1}^{2} R^{-2}\right)^{3 / 2}}=0 .
$$

Note that (21) is equivalent to

$$
\frac{d}{d x}\left\{R^{2}-\frac{1}{\sqrt{1+R^{\prime 2}+\Theta_{1}^{2} R^{-2}}}\right\}=0,
$$

so a first integral for (21) is given by

$$
R^{2}-\frac{1}{\sqrt{1+R^{\prime 2}+\Theta_{1}^{2} R^{-2}}}=I,
$$

where $I$ is an integration constant. The dynamics of (23) will therefore depend on two explicit parameters, $\Theta_{1}$ and $I$, as well as on the amplitude of the filament at the origin, $R(0)$. It is possible to find bounded periodic solutions $R(x)$ to (23). In order for such solutions to exist, we should restrict $I_{*}\left(\Theta_{1}\right)<I<I^{*}\left(\Theta_{1}\right)$, where $I_{*}\left(\Theta_{1}\right)$ and $I^{*}\left(\Theta_{1}\right)$ are constants that depend on the value of $\Theta_{1}$ taken. From the form of (23), we should have $I_{*}\left(\Theta_{1}\right)>-1$. On the other hand, assume that we have a bounded periodic solution satisfying $R_{*} \leq R(x) \leq R^{*}$, so that $R^{\prime}=0$ when either $R=R_{*}$ or $R=R^{*}$. Then, we must have

$$
R_{*}^{2}-\frac{1}{\sqrt{1+\Theta_{1}^{2} R_{*}^{-2}}}=I,
$$

therefore $I^{*}\left(\Theta_{1}\right)<R_{*}^{2}$.

It is perhaps easiest to view the dependence of a solution $R(x)$ on the constants $\Theta_{1}$ and $I$ by way of phase portraits, since these are useful for displaying periodic solutions. The cases for $\Theta_{1}=0$ and $\Theta_{1} \neq 0$ are qualitatively distinct. In particular, when $\Theta_{1}=0$, we obtain planar filaments which correspond to periodic $R(x)$ with $R_{\min }=-R_{\max }$, therefore $R(x)$ takes both positive and negative values - as seen in Fig. 1(a). In contrast, when $\Theta_{1} \neq 0$, we cannot have $R(x)=0$ for any $x$, else we have a division by zero. The result is that $R(x)$ remains positive for all $x$, and profiles for this case are demonstrated in Fig. 1(b). Note that there exists a single point at which $R^{\prime}(x) \equiv 0$ for all $x$ and $R(x)=R_{0}$, a constant. This point in the phase space corresponds directly to a helical filament with amplitude $R_{0}$.

We should remark that it is possible to solve (23) implicitly by separating variables. After appropriate manipulations, such a solution can be put into the implicit form:

$$
x= \pm \frac{1}{2} \int_{R_{*}^{2}-I}^{R(x)^{2}-I} \frac{\xi d \xi}{\sqrt{\left(4-\xi^{2}\right)(\xi+I)-\Theta_{1}^{2} \xi^{2}}} .
$$

This solution is not particularly enlightening, so numerical results shall be considered later for many different values of $\Theta_{1}$ and $I$. However, this formula does give one a way to calculate the period of 


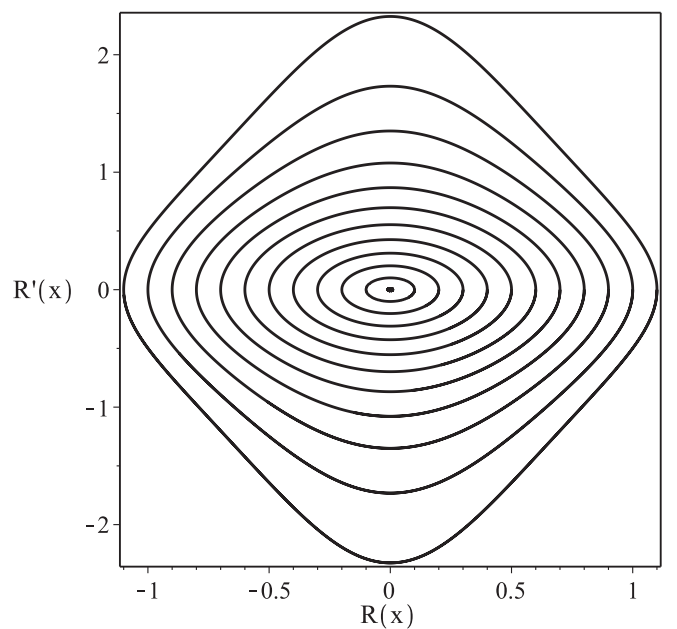

(a)

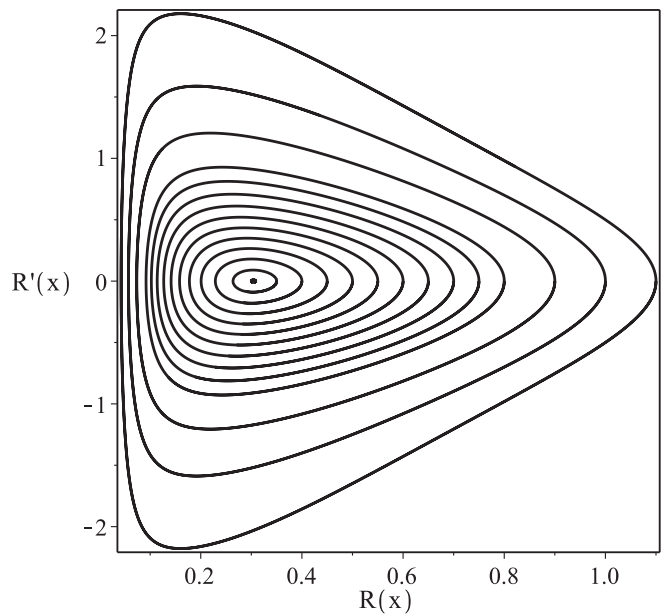

(b)

FIG. 1. Plots of the solutions $R(x)$ to (23) in the phase space $\left(R, R^{\prime}\right)$ when (a) $\Theta_{1}=0$, (b) $\Theta_{1}=0.1$. The helical filament corresponds to $\left(R, R^{\prime}\right)=(0.2517,0)$ and gives the minimal possible values of $J$, namely, $J=-0.8660$. The functions $R(x)$ corresponding to planar filaments are found in (a), while the functions $R(x)$ which are always positive are found in (b). This latter class of solutions corresponds to neither planar nor helical filaments; rather, such solutions correspond to generalized rotating filaments.

such a solution, assuming the upper and lower bounds on $R(x)$ are known. A period $T$ occurs when the solution trajectory goes from $R_{*}$ to $R^{*}$ and then back to $R_{*}$. Since Eq. (23) depends only on $R^{2}$ and not on $R$ explicitly, the solution should be symmetric in the sense that exactly half of the period occurs when the trajectory goes from $R_{*}$ to $R^{*}$. Therefore, we obtain the following formula for the period $T$ of the function $R(x)$ :

$$
T=\int_{R_{*}^{2}-I}^{R^{* 2}-I} \frac{\xi d \xi}{\sqrt{\left(4-\xi^{2}\right)(\xi+I)-\Theta_{1}^{2} \xi^{2}}} .
$$

We make one final interesting point before moving on to some specific cases. Recall that $\Theta^{\prime}(x)$ $=\Theta_{1} R(x)^{-2}$. If $R(x)$ is periodic with period $T$, then so is $\Theta^{\prime}(x)$. Further, one may show that the integral of a periodic function is periodic (and the period is preserved), therefore $\Theta(x)$ is periodic with period $T$. We then have that $\psi(x)$ is periodic whenever its amplitude $|\psi(x)|$ is periodic. This in turn implies that the filament is periodic in the space variable with respect to the $y$ and $z$ components, that is to say, $y(x+T, t)=y(x, t)$ and $z(x+T, t)=z(x, t)$ for all $x$ and all $t$. This makes sense, as the manner of motion is rotational.

\section{A. Small amplitude perturbations along a filament}

Let us introduce the functions $\zeta=R^{\prime} / R, \xi=\Theta^{\prime}$. We obtain the coupled system:

$$
\begin{aligned}
1+\frac{\zeta^{\prime}+\zeta^{2}-\xi^{2}}{1+R^{2}\left(\zeta^{2}+\xi^{2}\right)} & =0, \\
\xi^{\prime}+2 \zeta \xi & =0 .
\end{aligned}
$$

Assuming a small amplitude solution $|\psi|=|R| \ll 1$, say $|R| \approx \epsilon$ for a small parameter $\epsilon>0$, and noting that such an assumption does not imply that $\zeta$ is small, we obtain the simpler system:

$$
\begin{gathered}
\zeta^{\prime}+\zeta^{2}-\xi^{2}=-1, \\
\xi^{\prime}+2 \zeta \xi=0 .
\end{gathered}
$$


We find two classes of solutions to this transformed equation. The first family of solutions is parametrized by an arbitrary constant $a \in \mathbb{R}$ and reads

$$
\xi(x)=0 \quad \text { and } \quad \zeta(x)=-\tan (x+a) .
$$

Writing these in terms of $R$ and $\Theta$ gives

$$
R(x)=\frac{\epsilon}{\sqrt{1+\tan ^{2}(x+a)}} \text { and } \Theta(x)=\Theta_{0} .
$$

The profile for the vortex filament is then

$$
\mathbf{r}(x, t)=\left(x, \frac{\epsilon \cos \left(t-\Theta_{0}\right)}{\sqrt{1+\tan ^{2}(x+a)}},-\frac{\epsilon \sin \left(t-\Theta_{0}\right)}{\sqrt{1+\tan ^{2}(x+a)}}\right) .
$$

Note that this solution depends on three parameters: $a, \epsilon$, and $\Theta_{0}$.

The second class of solutions is also parametrized by an arbitrary constant $a \in \mathbb{R}$ and is given by

$$
\xi(x)=-\frac{8}{\sqrt{a^{2}+64}+a \cos (2 x)} \quad \text { and } \quad \zeta(x)=-\frac{1}{2} \frac{\xi^{\prime}(x)}{\xi(x)}=-\frac{a \sin (2 x)}{\sqrt{a^{2}+64}+a \cos (2 x)} .
$$

We find

$$
R(x)=\epsilon \sqrt{\sqrt{a^{2}+64}+a \cos (2 x)} \text { and } \Theta(x)=\Theta_{0}+\tan ^{-1}\left(\frac{\sqrt{a^{2}+64}-a}{8} \tan (x)\right) .
$$

The vortex filament is then described in the asymptotic limit by the formula:

$$
\begin{array}{r}
\mathbf{r}(x, t)=\left(x, \epsilon \sqrt{\sqrt{a^{2}+64}+a \cos (2 x)} \cos \left(\Theta_{0}-t+\tan ^{-1}\left(\frac{\sqrt{a^{2}+64}-a}{8} \tan (x)\right)\right),\right. \\
\left.\epsilon \sqrt{\sqrt{a^{2}+64}+a \cos (2 x)} \sin \left(\Theta_{0}-t+\tan ^{-1}\left(\frac{\sqrt{a^{2}+64}-a}{8} \tan (x)\right)\right)\right) .
\end{array}
$$

Again, this is a three-parameter family of vortex filament solutions.

The analytical solutions in the small amplitude regime given here are in good agreement with numerical solutions, which shall be discussed in Sec. III B. The first solution presented here corresponds to a rotation of the planar filament, due to the parameter $\Theta_{0}$. The second solution, however, is quite distinct from this. In fact, the solution (34) is a hybrid of the planar and helical solutions. The amplitude effectively behaves likes a planar filament, but the phase is space-variable. This is in contrast to the planar filament, which corresponds to space-independent phase. Meanwhile, the true helical filament has a constant amplitude and a linear dependence of the phase on the space variable $x$. So, the solution (34) is distinct from each of the two simpler cases. In terms of the phase portraits in Fig. 1, the family of solutions (30) behave like the solutions shown in Fig. 1(a), whereas the family of solutions (34) behave like those shown in Fig. 1(b).

\section{B. Numerical solutions and comparison with the analytical results}

In order to capture the behavior of the filaments corresponding to $w(x, t)$ with unconstrained amplitude and phase, we resort to numerical simulations of the coupled system of ordinary differential equations (18), obtaining numerical solutions for $R(x)$ and $\Theta(x)$. Taking the initial conditions $R(0)$ $=R_{0}, R^{\prime}(0)=0$ gives a periodic solution for $R$. Note that using $R^{\prime}(0) \neq 0$ does not change this fact: it simply translates the graph of $R$ along the $x$-axis. Likewise, we set $\Theta(0)=\Theta_{0}, \Theta^{\prime}(0)=\Theta_{1}$. The choice of $\Theta_{0}$ is not particularly interesting; however, by varying $\Theta_{1}$, we can obtain qualitatively different solutions. For $\Theta_{1}=0$, we obtain planar vortex filaments. However, as $\Theta_{1}$ increases, these solutions give way to more complex solution forms which become irregular in shape. As $\Theta_{1}$ increases further, the solutions become more like the regular helical solution. So, it seems that the 


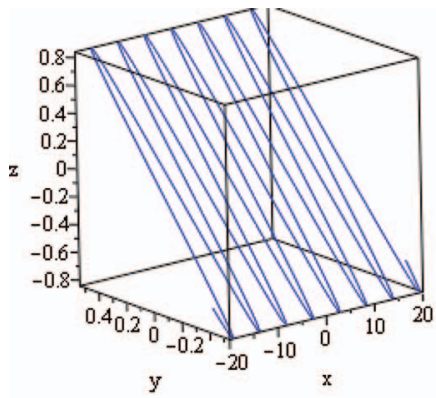

(a)

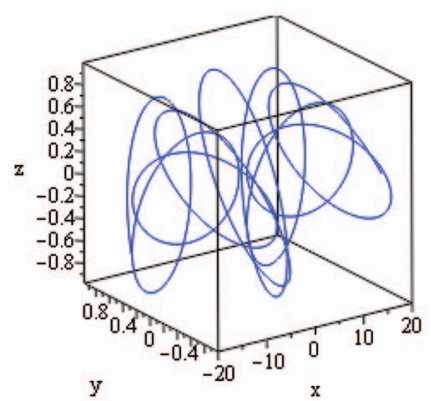

(d)

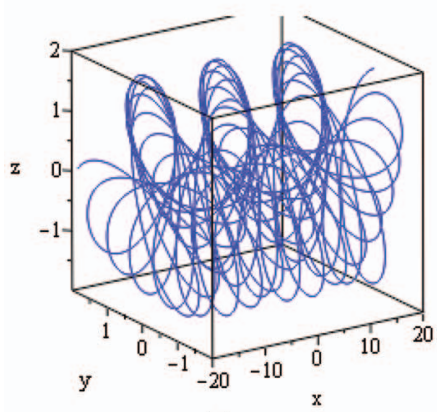

(g)

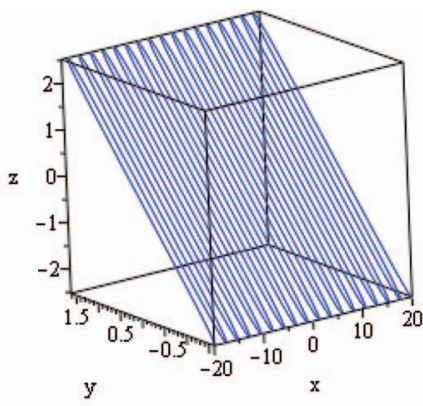

(b)

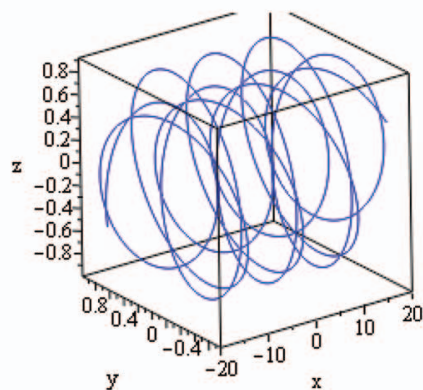

(e)

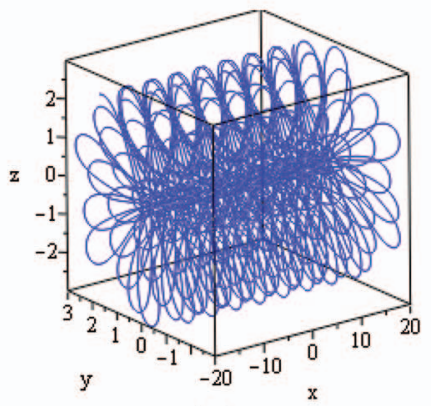

(h)

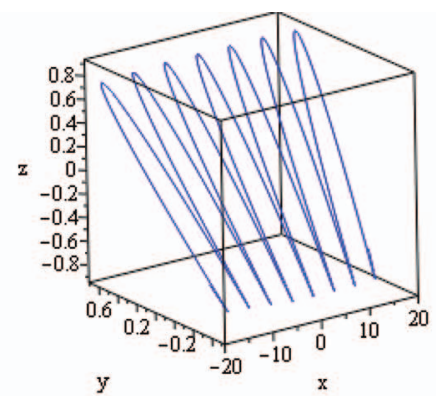

(c)

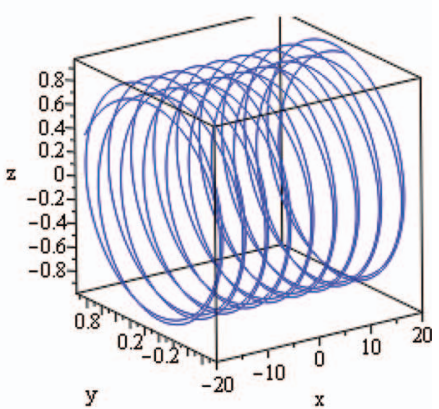

(f)

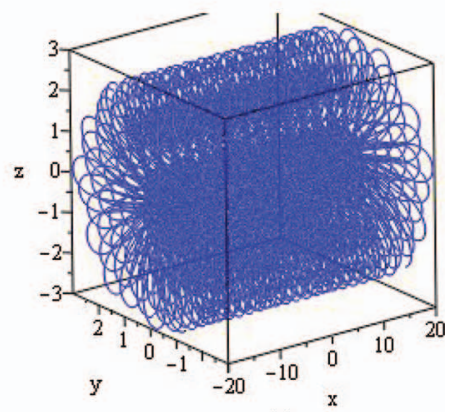

(i)

FIG. 2. Plots of the vortex filament solutions (16), when $R^{\prime}(0)=0, \Theta(0)=1$, and (a) $R(0)=1, \Theta^{\prime}(0)=0$; (b) $R(0)=3$, $\Theta^{\prime}(0)=0$; (c) $R(0)=1, \Theta^{\prime}(0)=0.05$; (d) $R(0)=1, \Theta^{\prime}(0)=0.5$; (e) $R(0)=1, \Theta^{\prime}(0)=1$; (f) $R(0)=1, \Theta^{\prime}(0)=2.5$; (g) $R(0)$ $=2, \Theta^{\prime}(0)=1.5$; (h) $R(0)=3, \Theta^{\prime}(0)=1.5$; (i) $R(0)=3, \Theta^{\prime}(0)=5$. Note that (a) and (b) depict planar filaments, while (f) depicts a perturbed helical filament. The filaments intermediate to these two are given in (c), (d), and (e). In particular, (c) demonstrates the bending of a planar filament when torsion increases. In (d) and (e), this planar filament gradually is deformed (through appropriate choice of parameters) into a helical filament, though (d) and (e) exhibit less regularity than either a planar or a helical filament. Finally, (g), (h), and (i) demonstrate more exotic behaviors which occur at larger amplitudes. These larger-amplitude filaments likely give way to superfluid turbulence.

planar vortex filaments and the helical vortex filaments are on two ends of a spectrum of solutions parametrized by $\Theta_{1}$.

In Fig. 2, we plot the filament solutions in the Cartesian reference frame for distinct model parameters. In Fig. 3, we provide a cross-section in the $y$-z-plane (directed along the $x$-axis), so as to more clearly depict the structures present in Fig. 2.

The numerical solution in the first panel is a planar vortex filament, with $\psi$ real-valued. Increasing $\Theta_{1}$, we find that the phase $\Theta(x)$ becomes increasingly linear, although periodic oscillations appear added to this linear trend. These oscillations are smoothed as $\Theta_{1}$ increases further. As $\Theta_{1}$ increases, and $\Theta(x)$ exhibits more of a linear trend, the solutions take on the form of helical filaments. 


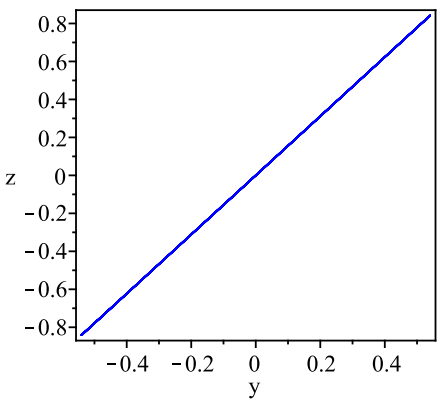

(a)

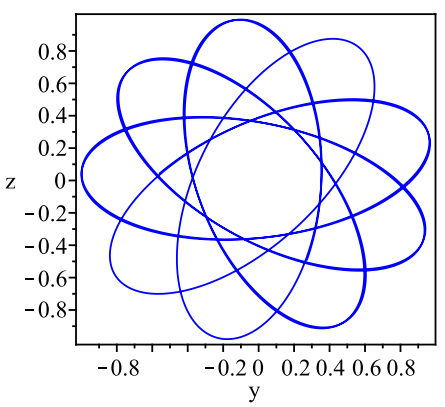

(d)

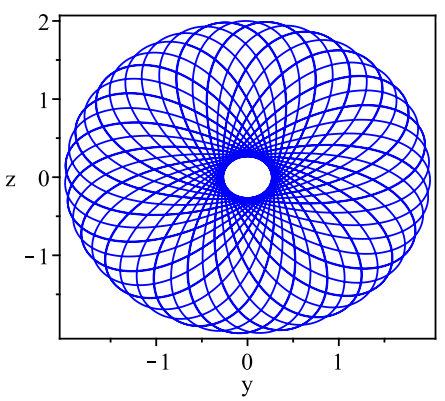

(g)

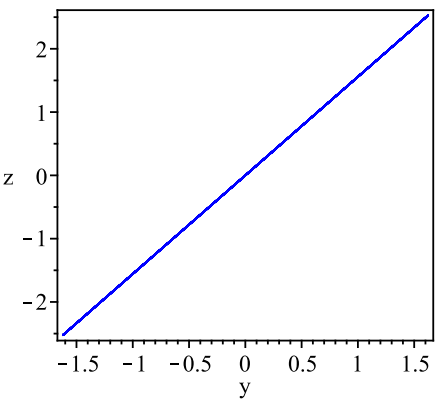

(b)

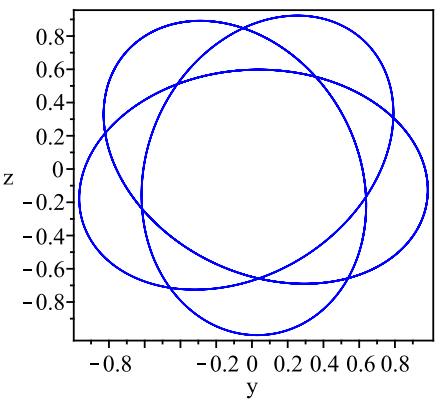

(e)

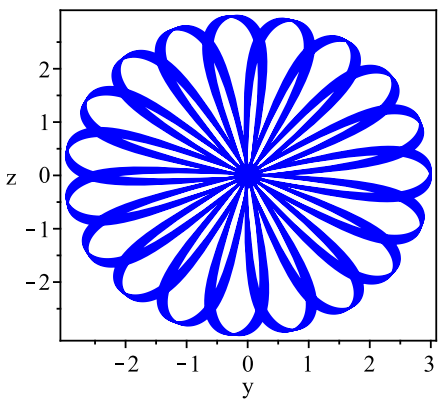

(h)

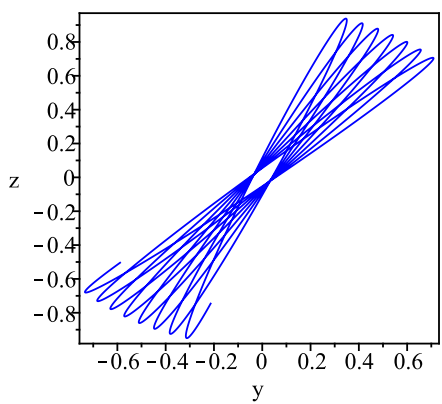

(c)

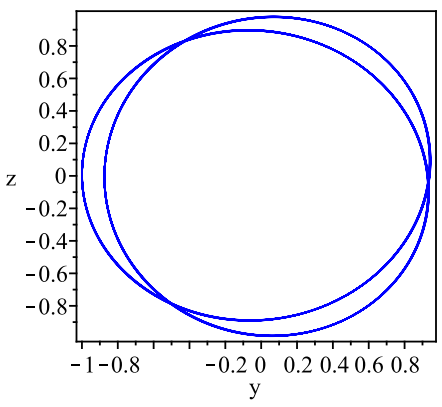

(f)

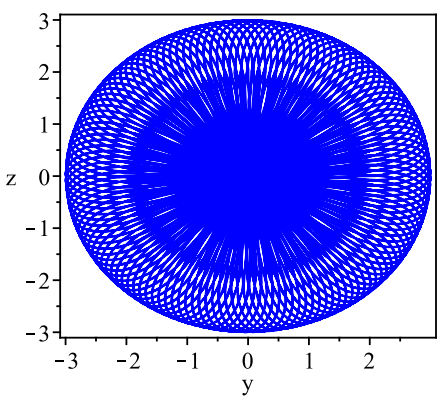

(i)

FIG. 3. Cross sections of the vortex filament solutions (16) in the $y$-z plane, when $R^{\prime}(0)=0, \Theta(0)=1$, and (a) $R(0)=1$, $\Theta^{\prime}(0)=0$; (b) $R(0)=3, \Theta^{\prime}(0)=0$; (c) $R(0)=1, \Theta^{\prime}(0)=0.05$; (d) $R(0)=1, \Theta^{\prime}(0)=0.5$; (e) $R(0)=1, \Theta^{\prime}(0)=1$; (f) $R(0)$ $=1, \Theta^{\prime}(0)=2.5$; $(\mathrm{g}) R(0)=2, \Theta^{\prime}(0)=1.5$; (h) $R(0)=3, \Theta^{\prime}(0)=1.5$; (i) $R(0)=3, \Theta^{\prime}(0)=5$. Though the three-dimensional plots of the filaments in Fig. 2 may appear unstructured in some cases, this view directed along the $x$-axis shows that all solutions exhibit at least some symmetry.

For the pure helical filament, $R(x)=R_{0}$ for all $x$, i.e., the amplitude is constant. As both amplitude and phase increase in value, the pure helical structure gives way to a filament which creates more intricate shapes. While the structures appear nearly chaotic, they actually are ordered, and exhibit certain symmetries.

The numerical results shown in panels (c) and (d), corresponding to those solutions which lie in between the planar and helical filaments, show clear agreement with the small amplitude results of Subsection III A, namely, the small amplitude solution (34). For this solution, the modulus of the potential function, $|R(x)|$, is strictly positive yet periodic, while the phase, $\Theta(x)$, is increasing yet exhibits an oscillatory behavior about a linear trend. Indeed, from the small amplitude solution (34), it is clear that the amplitude satisfies

$$
0<\epsilon \sqrt{\sqrt{a^{2}+64}-a} \leq R(x) \leq \epsilon \sqrt{\sqrt{a^{2}+64}+a} .
$$


This is in contrast to the planar filament, which changes signs on each period. Regarding the phase, from (34) we have that

$$
\Theta_{1}=\frac{\sqrt{a^{2}+64}-a}{8} \quad \text { or } \quad a=\frac{4\left(1-\Theta_{1}^{2}\right)}{\Theta_{1}} .
$$

Hence, the arbitrary constant $a$ in (34) is determined uniquely by the value of the parameter $\Theta_{1}$. If we expand $\Theta(x)$ found in (34) in a Taylor series in $x$, and make use of (36), we find

$$
\Theta(x)=\Theta_{0}+\Theta_{1} x+\Theta_{1}\left(1-\Theta_{1}^{2}\right)\left\{\frac{x^{3}}{3}+\frac{2-3 \Theta_{1}^{2}}{15} x^{5}+O\left(x^{7}\right)\right\} .
$$

So, in the small amplitude case, $\Theta_{1}=0$ corresponds exactly to the planar filament $\left(\Theta(x)=\Theta_{0}\right)$, while $\Theta_{1}=1$ corresponds to a helical case $\left(\Theta(x)=\Theta_{0}+x\right)$. For $0<\Theta_{1}<1$, we obtain the intermediate solutions shown numerically in parts (c) and (d) of Figures 1 and 2.

When $\psi$ takes the form of an amplitude function with constant phase, $\psi(x)=R(x) e^{-i \kappa}$, we obtain the planar vortex filament, along the lines studied by Hasimoto and others. ${ }^{9-11,16}$ If the initial conditions for this type of solution are varied, we see that the filaments will twist with increasing $x$ (this is best seen in Figs. 2(c) and 3(c)). We begin to see the emergence of solutions which are intermediate to the purely planar filaments (which rotate along the reference axis, yet remain on a plane) and the helical filaments (which form a rotating helical structure about the reference axis). Hence, the solution shown in Fig. 2(c) is a deformation of the Hasimoto solution to the LIA. This twisted planar filament is deformed further due to additional variations in the initial data, and it eventually becomes more helical in appearance.

When $\psi$ is a simple complex exponential $\left(\psi(x)=A e^{-i \kappa x}\right)$, we obtain filaments that form a helix about the reference axis. This helix rotates in a cork-screw manner as time increases. Such solutions were previously considered in the low temperature limit by Sonin. ${ }^{19}$ If such a $\psi$ is perturbed, we obtain a nearly helical filament which has been deformed in a manner which changes the periodicity of the filament in space. Such filaments are seen in the cross-section provided in Figs. 3(d)- 3(f). In particular, Fig. 2(f) represents a helical filament under a slight perturbation. In contrast, Figs. 3(e) and 3(d) demonstrate progressively greater deviations from the helical filament.

For larger amplitudes or sufficiently large initial change in the phase of $\psi$, we obtain solutions that become far more complicated in form. While many of these types of solutions, as shown in Figs. 2(g)-2(i), appear almost chaotic in form, the corresponding cross-section in Fig. 3 demonstrates that these solutions do exhibit order. Of course, unlike the planar or helical filaments, which have the possibility of being robust and maintaining their form in the face of structural perturbations, it is not likely that some of these more complicated structures would persist. More likely, vortex filaments with initial profiles matching some of these more complicated forms would likely give way to more complicated turbulence and reconnection events, due to excessive bending of the thin vortex. In this regime, non-local effects from the full Biot-Savart law (2) are likely to be needed.

\section{RELATION TO THE TORSION-CURVATURE FRAMEWORK}

Regarding other reference fames, since the motion of a self-induced vortex filament is often described in terms of curvature and torsion, we shall note how to recover these quantities from the Cartesian representation here. Using the Cartesian representation $\mathbf{r}(x, t)=(x, R(x) \cos (\Theta(x)$ $-t), R(x) \sin (\Theta(x)-t))$, one can obtain the curvature at a point $\mathcal{C}(x, t)$ and the torsion at a point $\mathcal{T}(x, t)$ using the standard formulas:

$$
\mathcal{C}(x, t)=\frac{\left|\mathbf{r}_{x} \times \mathbf{r}_{x x}\right|}{\left|\mathbf{r}_{x}\right|^{3}} \text { and } \mathcal{T}(x, t)=\frac{\left(\mathbf{r}_{x} \times \mathbf{r}_{x x}\right) \cdot \mathbf{r}_{x x x}}{\left|\mathbf{r}_{x} \times \mathbf{r}_{x x}\right|^{2}} .
$$

The explicit expressions, in terms of $R(x)$ and $\Theta(x)$, are too complicated to list here (they can be obtained with a computer algebra system, such as Maple or Mathematica, and would fill a page combined). While these expressions are rather complicated for arbitrary $R(x)$ and $\Theta(x)$, let us consider the special cases of planar and helical filaments. For the planar filament, $\Theta(x)=\Theta_{0}$, a 
constant, so we find

$$
\mathcal{C}(x, t)=\frac{\left|R^{\prime \prime}(x)\right|}{\left(1+R^{\prime 2}\right)^{3 / 2}} \quad \text { and } \quad \mathcal{T}(x, t)=0 .
$$

This is in agreement with what we know of the planar filament, in that it has zero torsion. As mentioned above, the deformation of the planar filament (obtained by perturbing the condition $\Theta(x)$ $=\Theta_{0}$ so that $\Theta(x)$ has a small $x$-dependent contribution) twists the filament, resulting in non-zero torsion. On the other hand, if instead we assume $R(x)=R_{0}$, the constant amplitude solution results in

$$
\begin{gathered}
\mathcal{C}(x, t)=\frac{\sqrt{R_{0}^{4} \Theta^{\prime 6}+R_{0}^{2} \Theta^{\prime 4}+R_{0}^{2} \Theta^{\prime \prime 2}}}{\left(1+R_{0}^{2} \Theta^{\prime 2}\right)^{3 / 2}}, \\
\mathcal{T}(x, t)=\frac{\Theta^{\prime 5}+3 \Theta^{\prime} \Theta^{\prime \prime 2}-\Theta^{\prime 2} \Theta^{\prime \prime \prime}}{R_{0}^{2} \Theta^{\prime 6}+\Theta^{\prime 4}+\Theta^{\prime \prime 2}} \cos (\Theta(x)-t)-\frac{\Theta^{\prime \prime}\left(2 \Theta^{\prime 3}+\Theta^{\prime \prime \prime}\right)}{R_{0}^{2} \Theta^{\prime 6}+\Theta^{\prime 4}+\Theta^{\prime \prime 2}} \sin (\Theta(x)-t) .
\end{gathered}
$$

In the case of a purely helical filament, $\Theta(x)=\kappa x$, and we have

$$
\mathcal{C}(x, t)=\frac{R_{0} k^{2}}{1+R_{0}^{2} k^{2}} \quad \text { and } \quad \mathcal{T}(x, t)=\frac{k}{1+R_{0}^{2} k^{2}} \cos (k x-t) .
$$

We see that the purely helical filament has positive constant curvature. Torsion varies depending on both $x$ and $t$ in a manner consistent with a helical curve.

\section{DISCUSSION AND CONCLUSIONS}

Physically, our results correspond to rotating vortex filaments which are open (the filaments do not cross) and remain within a finite distance from the reference axis. By varying two parameters, we have shown that there are essentially four classes of solutions. The most tame are planar and helical vortex filaments, and can be exactly described analytically. Note that the planar filament is the unique filament with space-variable curvature and zero torsion, while the pure helical filament has constant curvature and variable torsion. Intermediate to these two types of filaments are a class of solutions which merge properties of both types of filaments, and are essentially irregular helical filaments. These irregular helical filaments have variable curvature and torsion, which makes them much more complicated to solve analytically. In the small-amplitude regime, such filaments are approximated by the expression (34). Overall, these filaments are rather well-behaved and appear likely to persist.

Meanwhile, the fourth class of solutions occur at larger amplitudes or for more sharp increases of the phase of the stationary solutions. These solutions are more complicated and must be obtained numerically, since perturbation results are not useful in the intermediate amplitude regime. Some of these solutions are highlighted in Figs. 2(g)-2(i). While the solutions may appear disordered or chaotic, they actually do exhibit a form of regularity, as see by inspecting Fig. 3. Such solutions give us insight into the transition to strong turbulence in the Svistunov model we have considered here. Since the LIA approximates such turbulence, the cost is that such solutions exhibit far more regularity than might be expected. That said, these structures appear too intricate to persist in the case of superfluid friction (such as that included in the Hall-Vinen model), and these filaments would likely give way to turbulence in experiments. These filaments would also be likely candidates for single-filament reconnection events. This would occur when the vortex filament curves sharply, breaks, and the reconnects elsewhere along the filament. Of course, in terms of mathematics, such a solution would not be well-defined (it would be multivalued). To overcome this, one can introduce an alternate scaling $\mathbf{r}(x, t)=(f(x), y(x, t), z(x, t))$. Picking $f$ appropriately, one could obtain solutions along a curve as opposed to a straight line, which would permit a description of the intersecting filament solutions. This was considered for planar filaments perturbatively in Ref. 16

In addition to being useful for the study of the zero temperature limit of superfluid turbulence, the solutions to the 2D LIA (both analytical and numerical) are useful as a baseline of comparison 
for more complicated models. One such model is the full Biot-Savart law (2), which is complicated enough to mandate numerical solutions alone. Of course, the full Biot-Savart model of self-induced motion is non-local, whereas the LIA is a local model. As has previously been discussed, the LIA is most useful (and, in most agreement with the Biot-Savart law) in the case of strong turbulence. For weak turbulence, ${ }^{17}$ the LIA is much less useful, and other models may be considered, such as a truncated LIA that permits resonant wave interactions which are not present in the simplified LIA model. ${ }^{17}$ Understanding the time evolution of a vortex filament solution to the 2D LIA is also useful in that such a solution serves as a baseline of comparison for solution to the more complicated models which include superfluid friction parameters. Indeed, for $1 \gg \alpha>\alpha^{\prime}>0$, the solutions can be viewed as perturbations to the solutions presented here (at least in the rotating filament case).

It has been suggested ${ }^{34}$ that chaos is possible in some parameter regimes for the full HVBK model (including superfluid friction parameters). While this was for self-similar solutions, the same comment could of course be made for the HVBK model without the self-similarity restriction. Then, once the superfluid friction parameters are included (in the "warmer" temperature regime of about $1 \mathrm{~K}$ ), it is possible that the larger amplitude solutions here would give way to chaos. In principle, for small $\alpha$ and $\alpha^{\prime}$, the full HVBK model is a perturbation of the low-temperature LIA considered here. It would make sense, then, to consider a perturbation to the larger amplitude solutions, to account for $\alpha$ and $\alpha^{\prime}$, to first order. Such an analysis would cast light onto the stability of such solutions under small perturbations. Such small perturbations would likely either (i) cause instabilities that would grow into turbulence, or (ii) case decay of the filament due to the drag terms. In other words, we might expect the rotating filaments discussed here to either degenerate into a turbulent regime (non-integrable turbulence, more precisely) or to slowly decay into a line filament (so that small perturbations along the filament die off as time grows). This is a promising area of future work.

The stability or instability of the helical, planar, and soliton solutions under a variety of perturbations have been studied. Both analytical and numerical stability results exist for these classes of solutions; see Refs. 38-40 for some stability and instability results on helical filaments and arrays of helical filaments, Kida ${ }^{11}$ for results on the stability or instability of stationary states (such as solitons), and Van Gorder ${ }^{41}$ for results on orbital stability of the planar filament (a particular stationary state). Indeed, since the helical and planar solutions differ in their stability properties under a variety of perturbations, this suggests that the general stability properties of rotating filaments likely would not be understood using one technique. Helical solutions are unstable under many perturbations, whereas planar solutions can be shown to be orbitally stable. ${ }^{41}$ For the more complicated solutions shown here, not much is known, and it is likely that one would need to focus on specific cases in order to deduce the stability of a specific structure.

The stable structures would persist in time, and these would represent vortex filaments in regular superfluid flows. We can view the planar solutions as energy minimizing solutions, and these solutions maintain their form and are robust under small perturbations. On the other hand, the unstable structures could signify a variety of behaviors. Unstable solutions might collapse down to a line filament or they might amplify in time (the Donnelly-Glaberson instability ${ }^{42-44}$ ), and this occurs in the helical case when temperatures are positive (in the presence of mutual friction). Other types of unstable filaments might result in chaotic flows leading to turbulence. It is possible that the more exotic filaments found for larger amplitudes fall into this category, however this is not the only possibility. These complicated solutions are of larger amplitude and are more tightly coiled than the more tame solutions. Therefore, another possibility is that these solutions deamplify and uncoil, behaving like some of the more regular solutions at larger time scales after relevant perturbations are applied. Determining whether a given solution structure does one or the other (or, simply decays into a line filament) under perturbations is complicated in general, any must often be addressed in a case-by-case manner.

Furthermore, such structures arise due to the fact that we consider the zero-temperature limit (where mutual friction terms vanish). In this case, we have so-called eternal solutions (solutions which rotate without change in form for all time). If we include mutual friction terms, this creates dissipation. This dissipative effect may help to fight growth due to nonlinearity, meaning that for physically relevant scenarios, the more complicated solutions could persist for some timescales before amplifying and breaking apart or deamplifying into lower energy states. Therefore, even 
the more complicated shapes could be physically relevant in the sense that they might capture the small-time dynamics of more exotic filament configurations before the onset of reconnection and chaos occur at larger timescales.

\section{ACKNOWLEDGMENTS}

\section{R.A.V. was supported in part by NSF Grant No. 1144246.}

${ }^{1}$ H. E. Hall and W. F. Vinen, "The rotation of liquid helium II. I. Experiments on the propagation of second sound in uniformly rotating helium II," Proc. R. Soc. London A 238, 204 (1956).

${ }^{2}$ H. E. Hall and W. F. Vinen, "The rotation of liquid helium II. II. The theory of mutual friction in uniformly rotating helium II,” Proc. R. Soc. London A 238, 215 (1956).

${ }^{3}$ I. L. Bekarevich and I. M. Khalatnikov, "Phenomenological derivation of the equations of vortex motion in He II," Sov. Phys. JETP 13, 643 (1961).

${ }^{4}$ K. W. Schwarz, "Three-dimensional vortex dynamics in superfluid ${ }^{4} \mathrm{He}$ : Line-line and line-boundary interactions," Phys. Rev. B 31, 5782 (1985).

${ }^{5}$ L. D. Landau and E. M. Lifshitz, Fluid Mechanics (Addison and Wesley, 1959).

${ }^{6}$ R. J. Arms and F. R. Hama, "Localized-induction concept on a curved vortex and motion of an elliptic vortex ring," Phys. Fluids 8, 553 (1965).

${ }^{7}$ L. S. Da Rios, "Sul moto d'un liquido indefinite con un filetto vorticoso di forma qualunque," Rend. Circ. Mat. Palermo 22, 117 (1906).

${ }^{8}$ H. Hasimoto, “A soliton on a vortex filament," J. Fluid Mech. 51, 477 (1972).

${ }^{9}$ S. Kida, "A vortex filament moving without change of form," J. Fluid Mech. 112, 397 (1981).

${ }^{10}$ H. Hasimoto, "Motion of a vortex filament and its relation to elastica," J. Phys. Soc. Jpn. 31, 293 (1971).

${ }^{11}$ S. Kida, "Stability of a steady vortex filament," J. Phys. Soc. Jpn. 51, 1655 (1982).

${ }^{12}$ Y. Fukumoto, "Stationary configurations of a vortex filament in background flows," Proc. R. Soc. London A 453, 1205 (1997).

${ }^{13}$ M. Umeki, "A locally induced homoclinic motion of a vortex filament," Theor. Comput. Fluid Dyn. 24, 383 (2010).

${ }^{14}$ M. Umeki, "A real-space representation of a locally induced vortex filament," Theor. Appl. Mech. Jpn. 61, 195 (2013).

${ }^{15}$ R. A. Van Gorder, "Exact solution for the self-induced motion of a vortex filament in the arclength representation of the local induction approximation," Phys. Rev. E 86, 057301 (2012).

${ }^{16}$ R. A. Van Gorder, "Scaling laws and accurate small-amplitude stationary solution for the motion of a planar vortex filament in the Cartesian form of the local induction approximation," Phys. Rev. E 87, 043203 (2013).

${ }^{17}$ G. Boffetta, A. Celani, D. Dezzani, J. Laurie, and S. Nazarenko, "Modeling Kelvin wave cascades in superfluid helium," J. Low Temp. Phys. 156, 193 (2009).

${ }^{18}$ B. Svistunov, "Superfluid turbulence in the low-temperature limit," Phys. Rev. B 52, 3647 (1995).

${ }^{19}$ E. B. Sonin, "Dynamics of helical vortices and helical-vortex rings," EPL 97, 46002 (2012).

${ }^{20}$ B. K. Shivamoggi and G. J. F. van Heijst, "Motion of a vortex filament in the local induction approximation: Reformulation of the Da Rios-Betchov equations in the extrinsic filament coordinate space," Phys. Lett. A 374, 1742 (2010).

${ }^{21}$ R. A. Van Gorder, "Motion of a vortex filament in the local induction approximation: A perturbative approach," Theor. Comput. Fluid Dyn. 26, 161 (2012).

${ }^{22}$ R. A. Van Gorder, "Integrable stationary solution for the fully nonlinear local induction equation describing the motion of a vortex filament," Theor. Comput. Fluid Dyn. 26, 591 (2012).

${ }^{23}$ R. A. Van Gorder, "Motion of a helical vortex filament in superfluid ${ }^{4} \mathrm{He}$ under the extrinsic form of the local induction approximation," Phys. Fluids 25, 085101 (2013).

${ }^{24}$ N. Hietala and R. Hänninen, "Comment on 'Motion of a helical vortex filament in superfluid $4 \mathrm{He}$ under the extrinsic form of the local induction approximation' [Phys. Fluids 25, 085101 (2013)]," Phys. Fluids 26, 019101 (2014).

${ }^{25}$ R. A. Van Gorder, "Response to 'Comment on 'Motion of a helical vortex filament in superfluid 4He under the extrinsic form of the local induction approximation' [Phys. Fluids 26, 019101 (2014)]," Phys. Fluids 26, 019102 (2014).

${ }^{26}$ E. B. Sonin, "Symmetry of Kelvin-wave dynamics and the Kelvin-wave cascade in the $\mathrm{T}=0$ superfluid turbulence," Phys. Rev. B 85, 104516 (2012).

${ }^{27}$ R. E. Goldstein and D. M. Petrich, "Solitons, Euler's equation, and vortex patch dynamics," Phys. Rev. Lett. 69, 555 (1992).

${ }^{28}$ J. Langer and D. A. Singer, "Lagrangian aspects of the Kirchhoff elastic rod," SIAM Rev. 38, 605 (1996).

${ }^{29}$ Y. Fukumoto and M. Miyajima, "The localized induction hierarchy and the Lund-Regge equation," J. Phys. A 29, 8025 (1999).

${ }^{30}$ C. F. Barenghi and R. J. Donnelly, "Vortex rings in classical and quantum systems," Fluid Dyn. Res. 41(5), 051401 (2009).

${ }^{31}$ R. L. Ricca, D. C. Samuels, and C. F. Barenghi, "Evolution of vortex knots," J. Fluid Mech. 391, 29 (1999).

${ }^{32}$ M. Kursa, K. Bajer, and T. Lipniacki, "Cascade of vortex loops initiated by a single reconnection of quantum vortices," Phys. Rev. B 83(1), 014515 (2011).

${ }^{33}$ D. Kleckner and W. T. Irvine, "Creation and dynamics of knotted vortices," Nature Phys. 9(4), 253 (2013).

${ }^{34}$ T. Lipniacki, "Shape-preserving solutions for quantum vortex motion under localized induction approximation," Phys. Fluids 15, 1381 (2003).

${ }^{35}$ T. Lipniacki, "Quasi-static solutions for quantum vortex motion under the localized induction approximation," J. Fluid Mech. 477, 321 (2003).

${ }^{36}$ R. A. Van Gorder, "Self-similar vortex dynamics in superfluid ${ }^{4} \mathrm{He}$ under the Cartesian representation of the Hall-Vinen model including superfluid friction," Phys. Fluids 25, 095105 (2013). 
${ }^{37}$ R. A. Van Gorder, "Quantum vortex dynamics under the tangent representation of the local induction approximation," J. Fluid Mech. 740, 5 (2014).

${ }^{38}$ S. E. Widnall, "The stability of helical vortex filament," J. Fluid Mech. 54, 641 (1972).

${ }^{39}$ Y. Fukumoto and T. Miyazaki, "Three-dimensional distortions of a vortex filament with axial velocity," J. Fluid Mech. 222, 369 (1991).

${ }^{40}$ V. L. Okulov, “On the stability of multiple helical vortices," J. Fluid Mech. 521, 319 (2004).

${ }^{41}$ R. A. Van Gorder, "Orbital stability for rotating planar vortex filaments in the Cartesian and arclength forms of the local induction approximation,” J. Phys. Soc. Jpn. 82, 094005 (2013).

${ }^{42}$ D. K. Cheng, W. M. Cromar, and R. J. Donnelly, "Influence of an axial heat current on negative-ion trapping in rotating helium II," Phys. Rev. Lett. 31, 433 (1973).

${ }^{43}$ W. I. Glaberson, W. W. Johnson, and R. M. Ostermeier, "Instability of a vortex array in He II," Phys. Rev. Lett. 33, 1197 (1974).

${ }^{44}$ R. M. Ostermeier and W. I. Glaberson, "Instability of vortex lines in the presence of axial normal fluid flow," J. Low Temp. Phys. 21, 191 (1975). 